\title{
Temperature-Independent Multi-Parameter Measurement Based on a Tapered Bragg Fiber
}

\author{
Tiago J. M. Martins, Manuel B. Marques, Philippe Roy, Raphaël Jamier, Sébastien Février, and Orlando Frazão
}

\begin{abstract}
Temperature-independent strain and angle measurements are achieved resorting to a taper fabricated on a Bragg fiber using a $\mathrm{CO}_{2}$ laser. The characteristic bimodal interference of an untapered Bragg fiber is rendered multimode after taper fabrication and the resulting transmission spectra are analyzed as a function of strain, applied angle, and temperature variations. The intrinsic strain sensitivity exhibited by the Bragg fiber is increased 15 fold after tapering and reaches $22.68 \mathrm{pm} / \mu \varepsilon$. The angle and temperature measurements are also performed with maximum sensitivities of $185.10 \mathrm{pm} / \mathrm{deg}$ and $-12.20 \mathrm{pm} / \mathrm{K}$, respectively. The difference in wavelength shift promoted by variations in strain, angle, and temperature for the two fringes studied is examined. Strain and angle sensing with little temperature sensitivity is achieved, presenting a response of $2.87 \mathrm{pm} / \mu \varepsilon$ and $\mathbf{- 5 7 . 3 1} \mathrm{pm} / \mathrm{deg}$, respectively, for strain values up to $400 \mu \varepsilon$ and angles up to $10^{\circ}$. Simultaneous angle and strain measurements are demonstrated.
\end{abstract}

Index Terms-Optical fiber sensor, Bragg fiber, taper, interferometer.

\section{INTRODUCTION}

$\mathbf{O}$ PTICAL fiber technology has met many breakthroughs over the last decades, such as the development of more complex structures like Bragg fibers, whose theoretical model was proposed in 1978 [1]. These fibers are cylindrical waveguides consisting of a low-index core surrounded by concentric rings of material with alternating high and low refractive index, acting as a cylindrical Bragg mirror. As a result of this configuration, a bandgap transmission is expected [2], [3]. The range of applications spans a wide variety of possibilities, from high-power fiber lasers, since Bragg fibers allow the propagation of a single mode with a large effective area [4], to supercontinuum generation [5] and dispersion compensation [6]. In this case, the dispersion characteristics of Bragg fibers can be controlled by tailoring the periodic index modulation of the cladding.

Manuscript received February 15, 2016; revised March 24, 2016; accepted April 13, 2016. Date of publication April 20, 2016; date of current version May 16, 2016. This work was supported in part by the European Regional Development Fund through the Operational Programme for Competitiveness and Internationalisation-COMPETE 2020 Programme and in part by the National Funds through the Fundação para a Ciência e Tecnologia under Project POCI-01-0145-FEDER-006961

T. J. M. Martins, M. B. Marques, and O. Frazão are with the Instituto de Engenharia de Sistemas e Computadores, Tecnologia e Ciência, Porto 4200-465, Portugal, and also with the Physics and Astronomy Department, Faculty of Sciences, University of Porto, Porto 4099-002, Portugal (e-mail: tjmartins@fc.up.pt; mbmarque@fc.up.pt; ofrazao@fc.up.pt).

P. Roy, R. Jamier, and S. Février are with the Photonics Department, XLIM Research Institute, University of Limoges, Limoges 87060, France (e-mail: philippe.roy@xlim.fr; raphael.jamier@xlim.fr; sebastian.fevrier@xlim.fr).

Color versions of one or more of the figures in this letter are available online at http://ieeexplore.iee.org.

Digital Object Identifier 10.1109/LPT.2016.2555300
Optical sensing has also benefited from the introduction of Bragg fibers, allowing strain and temperature discrimination [7]. The underlying problem of cross-sensitivity that motivates the creative effort of designing new devices capable of physical parameter discrimination has resulted in several other proposals [8]-[10].

The potential of tapered structures in optical fibers as sensors has been thoroughly examined [11]-[13]. The fabrication of these devices has come to rely predominantly on $\mathrm{CO}_{2}$ lasers, given the high-power radiation delivered and the particular characteristics of the performed fiber heating [14], [15]. This leads to the possibility of obtaining different taper shapes [16].

This letter presents the evaluation of the behavior of a taper fabricated on a Bragg fiber, using a $\mathrm{CO}_{2}$ laser, as a sensor of strain, angle and temperature. In the $1550 \mathrm{~nm}$ region, the bimodal interference between the fundamental mode and a higher order mode exhibited by the untapered fiber is transformed in a multimode interference after taper fabrication. The interference conditions created within the tapered Bragg fiber are monitored as a function of strain, angle and temperature changes by interrogating the sensor in transmission.

\section{EXPERIMENTAL SETUP}

The proposed interferometric sensor is based on a taper fabricated on a $50 \mathrm{~mm}$-long stretch of hexagonal Bragg fiber resorting to a SYNRAD $48-1 \mathrm{CO}_{2}$ laser providing IR radiation with $10.6 \mu \mathrm{m}$ wavelength and $10 \mathrm{~W}$ maximum output power. The $50 \mathrm{~mm}$-long Bragg fiber is spliced at both ends with a standard singlemode fiber (SMF) and fixed onto AEROTECH motorized stages controlled via PC. The fabrication process consists of a double sweep of the optical fiber by the $\mathrm{CO}_{2}$ laser beam in order to reach smaller waist diameters. This strategy prevents considerable inconsistencies in the taper shape and damages to the optical medium. The two motorized stages move in the same direction with different speeds, set equal to $135 \mu \mathrm{m} / \mathrm{s}$ and $40 \mu \mathrm{m} / \mathrm{s}$, placing the fiber under additional mechanical stress. Laser power is $1.8 \mathrm{~W}$ and the taper length is chosen to be $5 \mathrm{~mm}$, yielding a $5 \mathrm{~mm}$ thin taper with a $35 \mu \mathrm{m}$ waist diameter. Fig. 1 (a) features one of the transition regions of the Bragg fiber taper, with a relatively smooth profile, which is important for power loss minimization and also to ensure some mechanical resistance to this kind of sensing device. The tapered Bragg fiber is interrogated in transmission using a broadband light source with a $1550 \mathrm{~nm}$ central wavelength and a $100 \mathrm{~nm}$ bandwidth as well as an ADVANTEST Q8384 Optical Spectrum Analyzer (OSA). The transmission spectra are studied setting the OSA resolution (res.) to $0.02 \mathrm{~nm}$. 


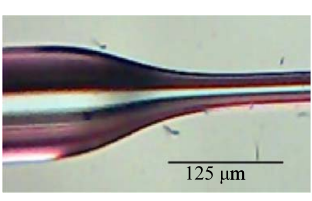

(a)

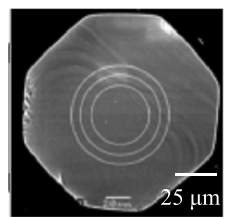

(b)

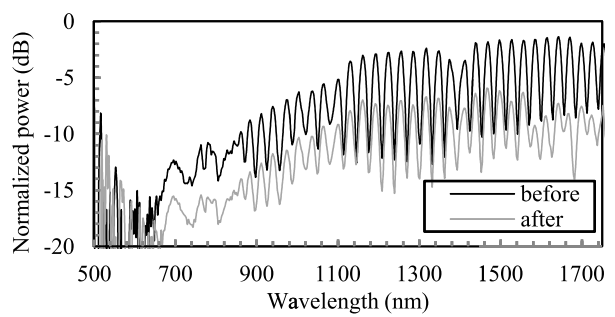

(d)

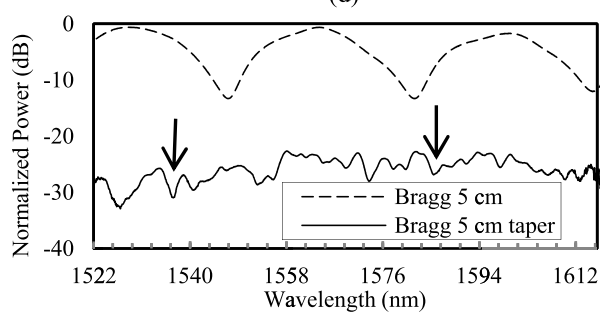

(e)

Fig. 1. Profile of the transition region of the fabricated $5 \mathrm{~mm}$ long taper (a); top view of both untapered (b) and tapered fibers (c); transmission spectra for these fibers in the ranges $\lambda \in[500 ; 1750] \mathrm{nm}($ OSA res $=10 \mathrm{~nm})(\mathrm{d})$ and $\lambda \in[1522 ; 1612] \mathrm{nm}$ (OSA res $=0.02 \mathrm{~nm})(\mathrm{e})$. The arrows identify the fringes monitored during strain characterization (for zero strain applied).

The intrinsic structure of the untapered Bragg fiber, consisting of a low-index core and a circularly periodic index modulation of the cladding, patent in Fig. 1 (b), creates a bimodal interference. This profile is kept almost unchanged after taper fabrication, except when it comes to its characteristic dimensions, considerably reduced: the three rings as well as the hexagonal border, considered just as an outer ring (circular profile), experience diameter reductions with ratios between $1: 3.3$ and $1: 3.6$, as observed in Fig. 1 (c). Fig. 1 (d) shows the transmission spectra for a wide wavelength range, between $500 \mathrm{~nm}$ and $1750 \mathrm{~nm}$, for the original and the tapered fibers, confirming that a bandgap-based transmission still takes place and that this bandgap still covers the 1550-nm region, even after tapering. The fabricated device still operates as a Bragg fiber. After taper fabrication, the interference becomes multimode as higher order modes are excited as seen in Fig. 1 (e). The detected average transmitted power is significantly decreased meaning that light is indeed being coupled from the core and inner ring to the outer rings, exciting higher order modes in the cladding. Light couples out from the core and inner ring at the taper transition region. In Fig. 1(d), the new interference fringes are not as clear as in Fig. 1 (e) given the used OSA resolution, equal to $10 \mathrm{~nm}$. In Fig. 1 (e) a higher resolution is chosen, equal to $0.02 \mathrm{~nm}$.

The experimental setup designed for sensor characterization is quite straightforward and besides the broadband light source and the spectrum analyzer it only includes an element responsible for introducing a perturbation to the Bragg fiber

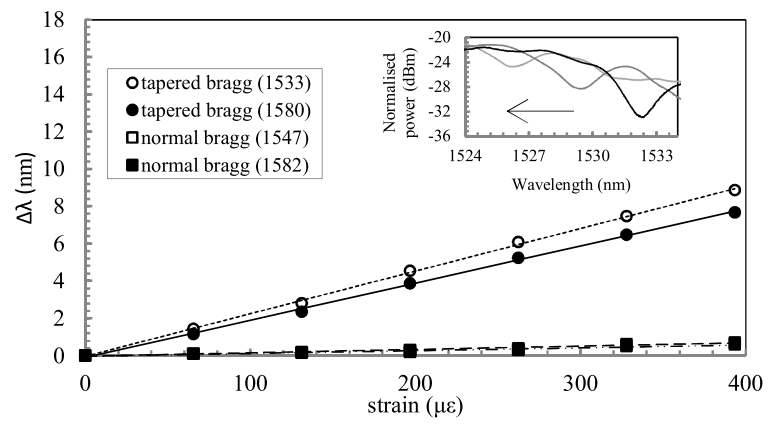

Fig. 2. Wavelength shift $\Delta \lambda$ as a function of the applied strain for both the tapered and the untapered Bragg fibers. Linear fits for all the considered fringes are displayed. Inset: shorter wavelength fringe evolution for increasing applied strain.

taper. To measure the wavelength shift induced by applying strain to the tapered portion of the fiber, a manually controlled translation stage is included to allow the variation of the applied stress by varying the distance between the two points where the fiber is fixed. The fiber is fixed on top of the very same translation stage and on a magnetized block placed at a certain distance. In the case of applied angle, a rotating platform with an uncertainty of $0.005 \mathrm{deg}$ is used. Temperature characterization of the tapered Bragg fiber follows a similar set of steps. The taper is placed inside an oven to allow raising its temperature up to $450{ }^{\circ} \mathrm{C}$.

\section{Results AnAlysis}

\section{A. Multi-Parameter Measurements}

The strain monitoring procedure is carried out by determining the location of two fringes in the interference spectrum of the tapered Bragg fiber, initially located at $\lambda_{1}=1536 \mathrm{~nm}$ and $\lambda_{2}=1584 \mathrm{~nm}$, as the applied strain increases. In order to determine whether the tapering process promotes a significant increase in the sensitivity of the device, the $50 \mathrm{~mm}$-long unchanged Bragg fibre is also monitored. The fringes considered are located at $\lambda_{1}=1547 \mathrm{~nm}$ and $\lambda_{2}=1582 \mathrm{~nm}$. The deviations concerning fringe position between both the tapered and untapered fibers are under $1 \%$, which is important if one wants a reasonable and conclusive sensitivity comparison. The graph in Fig. 2 clearly shows an increase in sensitivity as a result of the tapering process for both fringes. The calculated sensitivities are, from the shortest to the longest fringe wavelength, $22.68 \mathrm{pm} / \mu \varepsilon$ and $20.02 \mathrm{pm} / \mu \varepsilon$, for the tapered fiber, and $1.51 \mathrm{pm} / \mu \varepsilon$ and $1.78 \mathrm{pm} / \mu \varepsilon$, for the untapered fiber. The maximum increase is then of approximately 15 times and is registered for the fringe at shorter wavelengths. Increase in sensitivity as a consequence of the tapering process is expected given the mechanical properties of the fiber material. The tension $T$ applied through the fiber is obtained dividing the force $F$ by the cross-section area $A$, reduced by the tapering process. This means that the strain $\varepsilon=T / E=F /(A \times E)$, where $E$ is Young's modulus, increases despite applying the same force to the sensing device, resulting in larger sensitivities [17]. A rough estimate for the variation in strain, applying the same force, when 


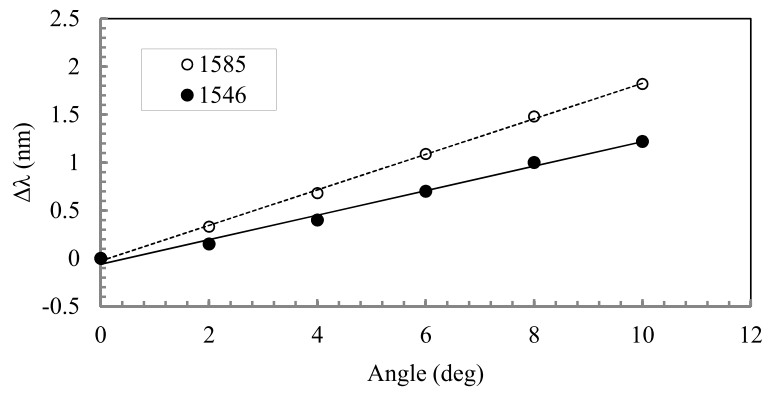

Fig. 3. Wavelength shift $\Delta \lambda$ as a function of the applied bend angle for the tapered Bragg fiber. Linear fits for both fringes are displayed.

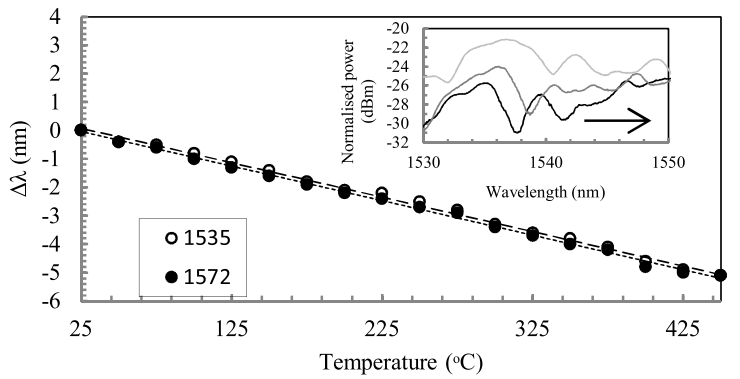

Fig. 4. Wavelength shift $\Delta \lambda$ as a function of the temperature for the tapered Bragg fiber. Linear fits for both fringes are displayed. Inset: shorter wavelength fringe evolution for increasing temperature.

one goes from the original fiber to the tapered one, can be attempted: $\varepsilon_{\text {taper }} / \varepsilon_{\text {fibre }}=\left(d_{\text {fibre }} / d_{\text {taper }}\right)^{2}=(125 / 35)^{2} \approx 13$, where $d$ is the diameter. This estimate is not far from the variations in sensitivity obtained experimentally. Deviations from this prediction, resulting in lower or larger sensitivity ratios between tapered and untapered fibers, can be due to the different spatial profiles of the excited modes.

The taper exhibits considerable curvature sensitivity in the studied range of applied bend angles, from 0 to $10 \mathrm{deg}$, for fringes located at $\lambda_{1}=1546 \mathrm{~nm}$ and $\lambda_{2}=1585 \mathrm{~nm}$, as shown in Fig. 3. The sensitivities are, from the shortest to the longest fringe wavelength, $127.81 \mathrm{pm} / \mathrm{deg}$ and $185.10 \mathrm{pm} / \mathrm{deg}$. The calculated values for these strain and angle sensitivities surpass those presented in some of the literature [6]-[9], [11], though verified over a smaller range of applied strain and angle.

Concerning temperature characterization, the examined fringes are initially located at $\lambda_{1}=1535 \mathrm{~nm}$ and $\lambda_{2}=1572 \mathrm{~nm}$ and the promoted wavelength shifts due to changing temperature are registered and displayed in Fig. 4. The sensitivities are, from the shortest to the longest fringe wavelength, $-12.11 \mathrm{pm} / \mathrm{K}$ and $-12.20 \mathrm{pm} / \mathrm{K}$, also larger than some reported results [6], [7]. Special attention is taken not to have large deviations in fringe locations considered for strain, angle and temperature measurements, resulting in a maximum deviation under $1 \%$.

\section{B. Temperature-Insensitive Measurements}

The difference between fringe sensitivities is larger in the case of applied strain and angle. The study of the difference in fringe location $\lambda_{2}-\lambda_{1}$ makes the possibility of strain and

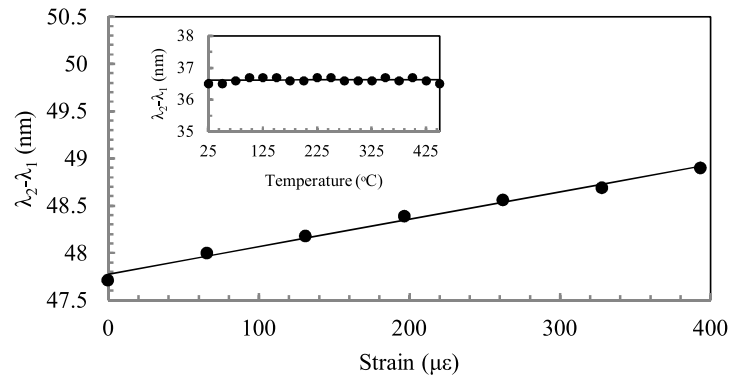

Fig. 5. Difference in fringe location $\lambda_{2}-\lambda_{1}$ as a function of the applied strain for the tapered Bragg fiber. Inset: difference in fringe location $\lambda_{2}-\lambda_{1}$ as a function of the temperature for the tapered Bragg fiber. Linear fits for both graphics are displayed.

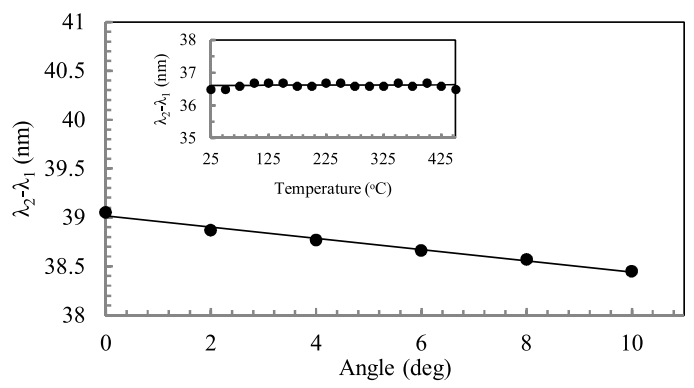

Fig. 6. Difference in fringe location $\lambda_{2}-\lambda_{1}$ as a function of the applied angle for the tapered Bragg fiber. Inset: difference in fringe location $\lambda_{2}-\lambda_{1}$ as a function of the temperature for the tapered Bragg fiber. Linear fits for both graphics are displayed.

angle sensing with little temperature sensitivity apparent as observed through Figs. 5 and 6. These experimental results agree well with our early conjectures: only the perturbations that change the geometry of this device, such as strain and applied angle, are expected to give rise to different sensitivities for different interference fringes. Taking applied angle as an example, a guided mode confined to the surroundings of the fiber core will experience a different stress resulting from curvature than another one extending far into the cladding. This results in different bend angle sensitivities when one monitors fringes corresponding to interference involving either one of these modes. In the case of temperature, given the diffusivity of silica, equal to $0.7 \mathrm{~mm}^{2} / \mathrm{s}$ [13], heat should reach the core of a $35 \mu \mathrm{m}$ waist diameter taper in less than $2 \mathrm{~ms}$. Thus, the modes propagating along the taper should experience approximately the same change in temperature, resulting in fringe sensitivities close to each other. In fact, the new sensitivities, obtained resorting to this sensing scheme and equal to $2.87 \mathrm{pm} / \mu \varepsilon,-57.31 \mathrm{pm} / \mathrm{deg}$ and $-0.04 \mathrm{pm} / \mathrm{K}$, show that strain and angle monitoring can be performed with sensitivities approximately 70 and 1400 times larger than the one associated to temperature. Through a different perspective, the variations in strain and angle due to temperature changes are around $0.01 \mu \varepsilon / \mathrm{K}$ and $0.0007 \mathrm{deg} / \mathrm{K}$. Considering the maximum temperature achieved during the characterization of this sensor, equal to $450{ }^{\circ} \mathrm{C}$, the variations in strain and angle are $4.5 \mu \varepsilon$ and $0.3 \mathrm{deg}$, representing, respectively, $1 \%$ and $3 \%$ of the maximum values for applied strain and angle. 


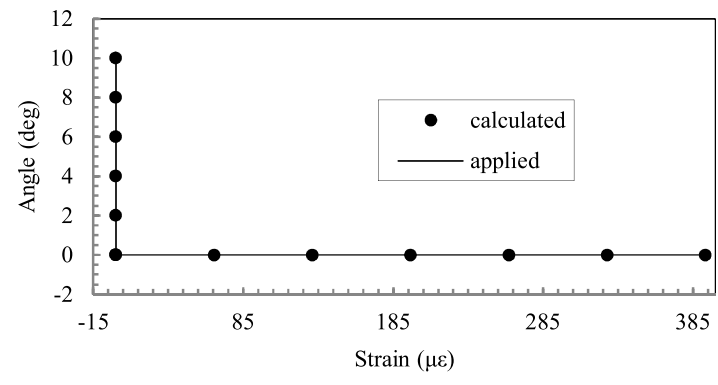

Fig. 7. Sensor output as determined by (1) for applied angle and strain.

\section{Simultaneous Strain and Angle Measurement}

The analysis of the experimental data collected reveals that for all the fringes under evaluation a good linear behavior is obtained, supported by the fact that all determination coefficients $\mathrm{R}^{2}$ are larger than 0.99 . The possibility of considering simultaneous strain and angle measurement is reinforced by the fact that the strain and angle sensitivities obtained through the sensing scheme described in the previous section have different signs: the difference $\lambda_{2}-\lambda_{1}$ increases for increasing strain and decreases for increasing angle. Thus, cross-sensitivity can be compensated. Using the sensitivities calculated from the analysis of the experimental data in Figs. 2 and 3, one can write the angle $\theta$ and the strain $\varepsilon$ as functions of the wavelength shifts $\Delta \lambda_{1}$ and $\Delta \lambda_{2}$

$$
\left[\begin{array}{l}
\varepsilon \\
\theta
\end{array}\right]=\frac{1}{1639.3}\left[\begin{array}{cc}
185.10 & -127.81 \\
-20.02 & 22.68
\end{array}\right]\left[\begin{array}{l}
\Delta \lambda_{1} \\
\Delta \lambda_{2}
\end{array}\right] \text {. }
$$

The set of equations (1) allows the calculation of the angle and strain using the wavelength shifts already determined experimentally. Strain measurements were performed between 0 and $400 \mu \varepsilon$ without any angle applied, while angle measurements were performed between 0 and 10 deg without any stress applied as shown in Fig. 7. From these results, root-meansquare deviations of $\pm 0.02 \mu \varepsilon$ and \pm 0.004 deg were obtained for strain and angle measurements, respectively.

\section{CONCLUSION}

The $\mathrm{CO}_{2}$ laser-supported tapering process changes the spatial profile of a Bragg fiber altering the interference created inside the fiber, moving from a bimodal to a multimode interference as light couples from the core and inner ring to excite higher order cladding modes. The resulting changes in strain sensitivity are remarkable with values reaching $22.68 \mathrm{pm} / \mu \varepsilon$, which represents an increase of 15 times the sensitivity of an untapered Bragg fiber. Angle measurements are performed with maximum sensitivity of $185.10 \mathrm{pm} / \mathrm{deg}$. The obtained temperature sensitivities, reaching $-12.20 \mathrm{pm} / \mathrm{K}$, are also worth mentioning, along with the fact that the range of temperature values covered goes from $25^{\circ} \mathrm{C}$ up to $450{ }^{\circ} \mathrm{C}$. Temperature-independent strain and angle sensing based on the same tapered Bragg fiber but employing a different sensing scheme, relying on the inspection of the difference between the studied fringes locations, is also proposed with sensitivities equal to $2.87 \mathrm{pm} / \mu \varepsilon$ and $-57.31 \mathrm{pm} / \mathrm{deg}$. These values are still larger than some of the reported in the literature. Strain and angle variations due to changes in temperature are equal to $0.01 \mu \varepsilon / \mathrm{K}$ and $0.0007 \mathrm{deg} / \mathrm{K}$, respectively. Simultaneous angle and strain measurement is also demonstrated.

\section{ACKNOWLEDGMENTS}

The authors acknowledge S. L. Semjonov, M. E. Likhachev, M. M. Bubnov, and E. M. Dianov from the Fiber Optics Research Center, Moscow, and V. F. Khopin, M. Y. Salganskii, and A. N. Guryanov from the Institute of Chemistry of High Purity Substances, and N. Novgorod for the fabrication of the fiber samples.

\section{REFERENCES}

[1] P. Yeh, A. Yariv, and E. Marom, "Theory of Bragg fiber," J. Opt. Soc. Amer, vol. 68, no. 9, pp. 1196-1201, Sep. 1978.

[2] T. Katagiri, Y. Matsuura, and M. Miyagi, "All-solid single-mode Bragg fibers for compact fiber devices," J. Lightw. Technol., vol. 24, no. 11, pp. 4314-4318, Nov. 2006.

[3] L. Ma and Y. Matsuura, "Bragg fiber taper for subwavelength beam generation," J. Lightw. Technol., vol. 26, no. 24, pp. 3847-3852, Dec. $15,2008$.

[4] S. Février et al., "Very large effective area singlemode photonic bandgap fibre," Electron. Lett., vol. 39, no. 2, pp. 1240-1242, Aug., 2003.

[5] R. Jamier, N. Ducros, S. Février, M. E. Likhachev, and M. Y. Salganskii, "Tight control of the spectral broadening obtained by nonlinear conversion in a photonic bandgap fiber," in Proc. CLEO/IQEC, Jun. 2009, pp. 1-2, paper JWA53.

[6] F. Gérôme et al., "Highly dispersive large mode area photonic bandgap fiber," Opt. Lett., vol. 32, no. 10, pp. 1208-1210, May 2007.

[7] O. Frazão, L. M. N. Amaral, J. M. Baptista, P. Roy, R. Jamier, and S. Février, "Strain and temperature discrimination using modal interferometry in Bragg fibers," IEEE Photon. Technol. Lett., vol. 22, no. 21, pp. 1616-1618, Nov. 1, 2010.

[8] S. Février, M. A. Melo, P. V. S. Marques, and J. L. Santos, "Chirped Bragg grating fabricated in fused fibre taper for strain-temperature discrimination," Meas. Sci. Technol., vol. 16, no. 4, pp. 984-988, Mar. 2005.

[9] O. Frazão, L. Marques, J. M. Marques, J. M. Baptista, and J. L. Santos, "Simple sensing head geometry using fibre Bragg gratings for straintemperature discrimination," Opt. Commun., vol. 279, no. 1, pp. 68-71, Jun. 2007.

[10] S. Rota-Rodrigo, M. López-Amo, J. Kobelke, K. Schuster, J. L. Santos, and O. Frazão, "Multimodal interferometer based on a suspended core fiber for simultaneous measurement of physical parameters," J. Lightw. Technol., vol. 33, no. 12, pp. 2468-2473, Jun. 15, 2015.

[11] W. Du, X. Tao, and H.-Y. Tam, "Temperature independent strain measurement with a fiber grating tapered cavity sensor," IEEE Photon. Technol. Lett., vol. 11, no. 5, pp. 596-598, May 1999.

[12] J. Villatoro, V. P. Minkovich, and D. Monzón-Hernández, "Temperatureindependent strain sensor made from tapered holey optical fiber," Opt. Lett., vol. 31, no. 3, pp. 305-307, Feb. 2006.

[13] J. P. Moura et al., "Optical inclinometer based on a phase-shifted Bragg grating in a taper configuration," IEEE Photon. Technol. Lett., vol. 26 , no. 4, pp. 405-407, Feb. 15, 2014.

[14] F. Bayle and J.-P. Meunier, "Efficient fabrication of fused-fiber biconical taper structures by a scanned $\mathrm{CO}_{2}$ laser beam technique," Appl. Opt., vol. 44, no. 30, pp. 6402-6411, Oct. 2005.

[15] S. T. Yang, M. J. Matthews, S. Elhadj, V. G. Draggoo, and S. E. Bisson, "Thermal transport in $\mathrm{CO}_{2}$ laser irradiated fused silica: In situ measurements and analysis," J. Appl. Phys., vol. 106, no. 10, pp. 103106-1-103106-7, Jul. 2009.

[16] T. E. Dimmick, G. Kakarantzas, T. A. Birks, and R. St. J. Russell, "Carbon dioxide laser fabrication of fused-fiber couplers and tapers," Appl. Opt., vol. 38, no. 33, pp. 6845-6848, Nov. 1999.

[17] O. Frazão, S. F. O. Silva, A. Guerreiro, J. L. Santos, L. A. Ferreira, and F. M. Araújo, "Strain sensitivity control of fiber Bragg grating structures with fused tapers," Appl. Opt., vol. 46, no. 36, pp. 8578-8582, Dec. 2007. 